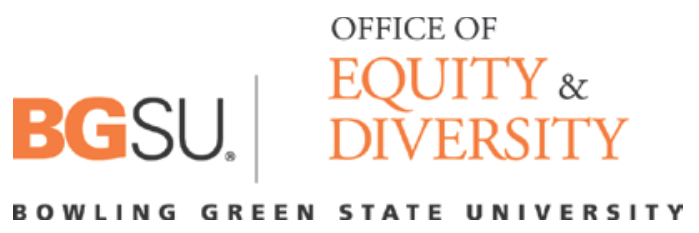

\title{
Facilitated Conversation
}

The Office of Equity and Diversity offers facilitated conversations as a means of resolution. Facilitated conversations provide a voluntary process in which a facilitator guides participants in reaching acceptable solutions to the issues they face. The process aims neither to erase what happened in the past, nor to determine who is right or wrong. It provides individuals with an opportunity to step back and engage in a respectful and confidential discussion where they can, for example:

- Re-establish trust and engage in meaningful communication;

- Speak freely and openly about their needs, interests and opinions, and understand the other's point of view;

- $\quad$ Propose viable and lasting solutions to a conflict; and

- Negotiate the terms of a verbal commitment, agreement or resolution.

\section{What is a facilitated conversation?}

The process of facilitated conversations is future-focused. Facilitated conversations are considered more informal and are used most often to restore interpersonal workplace/educational relationships. A facilitated conversation often ends with a verbal commitment whereby the parties agree to make specific changes in order to resolve the issue and ensure the conflict does not resurface.

\section{What happens when I decide to go ahead with a facilitated conversation?}

Typically, you would decide to go ahead with a facilitated conversation shortly after the consultation phase. At this point, the OED Officer helps determine the best way to invite the other party(ies) into the process. The other party(ies) will then meet or speak privately with the OED Officer, just as you did, and the OED Officer will explore the various options. If the other parties are interested in going ahead with a facilitated conversation our office will proceed to the next step.

\section{What happens when both parties have agreed to proceed?}

Each situation is unique, but once the parties agree to proceed with a facilitated conversation, our office staff will set a time and date that a meeting will take place and the parties will be given information as to where to attend the facilitated conversation. Participants should be prepared as to what they would like to discuss at the facilitated conversation. The facilitator's goal is to ensure that the participants are ready to listen, understand and collaborate with one another.

\section{What happens during the facilitated conversation?}

The parties meet on an agreed-upon date to engage in a process led by the facilitator. First, the ground rules are set to establish a respectful environment, and the parties then have an opportunity to share their experience of the situation without interruption. Next, core issues are defined and prioritized, and the parties engage in a 
dialogue. Each issue is explored through an interest-based lens which encourages curiosity, active listening, creativity and active problem solving. These conversations can at times be challenging and the facilitator's role is to assist the parties in their efforts to hold a respectful and productive conversation.

When resolution is reached for each issue, the parties acknowledge their commitments verbally, or sign an agreement. The agreement is written and executed by the parties and it details their agreed-upon solutions. The Office of Equity and Diversity may follow-up with the parties. If, for any reason, the situation has not improved, the parties have the option to engage the Office of Equity and Diversity in a formal process.

\section{What happens if the process is not successful?}

In most cases, a resolution can be reached. However, in some instances, the parties only reach a partial agreement or more rarely, no agreement is reached. In these cases, the parties still have access to formal resolution mechanisms through the Office of Equity and Diversity's complaint procedures found at http://www.bgsu.edu/offices/oed/page115857.html . 